\title{
Decision making under measure-based granular uncertainty with intuitionistic fuzzy sets
}

\author{
Yige Xue $^{1} \cdot$ Yong Deng ${ }^{1,2}$ (D) \\ Accepted: 13 January 2021 / Published online: 5 February 2021 \\ (C) The Author(s), under exclusive licence to Springer Science+Business Media, LLC part of Springer Nature 2021
}

\begin{abstract}
Yager has proposed the decision making under measure-based granular uncertainty, which can make decision with the aid of Choquet integral, measure and representative payoffs. The decision making under measure-based granular uncertainty is an effective tool to deal with uncertain issues. The intuitionistic fuzzy environment is the more real environment. Since the decision making under measure-based granular uncertainty is not based on intuitionistic fuzzy environment, it cannot effectively solve the decision issues in the intuitionistic fuzzy environment. Then, when the issues of decision making are under intuitionistic fuzzy environment, what is the decision making under measure-based granular uncertainty with intuitionistic fuzzy sets is still an open issue. To deal with this kind of issues, this paper proposes the decision making under measure-based granular uncertainty with intuitionistic fuzzy sets. The decision making under measure-based granular uncertainty with intuitionistic fuzzy sets can effectively solve the decision making issues in the intuitionistic fuzzy environment, in other words, it can extend the decision making under measure-based granular uncertainty to the intuitionistic fuzzy environment. Numerical examples are applied to verify the validity of the decision making under measure-based granular uncertainty with intuitionistic fuzzy sets. The experimental results demonstrate that the decision making under measure-based granular uncertainty with intuitionistic fuzzy sets can represent the objects successfully and make decision effectively. In addition, a practical application of applied intelligence is used to compare the performance between the proposed model and the decision making under measure-based granular uncertainty. The experimental results show that the proposed model can solve some decision problems that the decision making under measure-based granular uncertainty cannot solve.
\end{abstract}

Keywords Measure · Decision making · Granular uncertainty · Intuitionistic fuzzy sets · Applied intelligence

\section{Introduction}

In the real world, there are plenty of uncertainties [1], and many issues about uncertainty [2]. To address those situations, many mathematical theories and models are proposed. Pan et al. [3] combined the fuzzy sets and Dempster-Shafer evidence theory to improve the bayesian network and applied it to analysis risk. Gao and Deng [4] proposed the Pseudo-Pascal triangle of maximum Deng

Yong Deng

dengentropy@uestc.edu.cn

1 Institute of Fundamental and Frontier Science, University of Electronic Science and Technology of China, Chengdu, 610054, China

2 School of Eduction, Shaanxi Normal University, Xi' an, 710062, China entropy. Fujita et al. [5] proposed a temporal-spatial composite sequential approach under granular computing environment. Athira et al. [6] proposed a new entropy model with the aid of Pythagorean fuzzy soft sets. Mohd et al. [7] extended the application scope of Pythagorean fuzzy sets into the green supplier development field. Jiang et al. [8] proposed a novel network model, which can combine bayesian network with Z-number.

Among these algorithms and theories, the intuitionistic fuzzy sets is an extent of fuzzy sets, which consist of intuitionistic fuzzy numbers $[9,10]$. It means that intuitionistic fuzzy sets is made up of membership degree, nonmembership degree and hesitancy degree, which is more flexible than fuzzy sets in representing the uncertainties. When it is not sure whether the value belongs to membership degree or non-membership degree, it is considered hesitancy degree, which gives more scope to represent the uncertainty. Relying on the advantages on indicating vague 
information, the intuitionistic fuzzy sets has been applied on many fields widely by scholars at home and abroad [11]. Wang et al. [12] proposed an new reasoning method, which is based on the intuitionistic fuzzy sets. Garg and Rani [13] found not only an new ranking method but also some novel aggregation operators under complex intuitionistic fuzzy environment. Zhou and $\mathrm{Xu}$ [14] analyzed the envelopment, fused preference and improved the membership of intuitionistic fuzzy sets. Alcantud et al. [15] proposed a new method to aggregate of infinite chains of intuitionistic fuzzy sets. Ngan et al. [16] applied the quaternion numbers to indicate complex intuitionistic fuzzy sets. Among all the applications of intuitionisitic fuzzy sets, the most representative one is the application of intuitionisitic fuzzy sets in decision making. Song et al. [17] improve the intuitionistic fuzzy sets with the aid of divergence-based cross entropy to address the issues of decision making. Son et al. [18] proposed a new representation of intuitionistic fuzzy sets and applied it to solve critical decision making. Garg and Singh [19] applied the similarity measurement theory and intuitionistic fuzzy sets to solve decision making problems. Meng et al. [20] introduced the linguistic intuitionistic fuzzy preference relations and applied it to address the multi-criteria decision making issues. Zhou and $\mathrm{Xu}$ [21] extended the intuitionistic fuzzy sets and applied it to deal with issues of decision making with risk preference.

When the space of uncertain variables is large, how to express uncertain variables is a very time-consuming and laborious problem. When uncertain variables are evaluated in large space, we can simplify the knowledge acquisition process by using space granulation. This process of simplifying knowledge acquisition is also very beneficial for decision-making. Then, using granulation to simplify knowledge acquisition can introduce inaccuracies into the relevant decision making process. Recently, Yager proposed the decision making under measure-based granular uncertainty [22], which has promising aspects. The decision making under measure-based granular uncertainty is a very flexible and efficient way to make decision, which represents uncertain information in the granulation of space, and potential uncertainty is generally expressed as measure. In this way, the space of the issues of decision making and granulation of this space need be determined. Then the measure corresponding to the granulation is given. Hence, analyze the actual problem and give the payoffs of each candidate solution. Then the payoff bags of the issue according to payoffs and granulation will be calculated. Hence, the corresponding representative payoff from each payoff bag will be obtained, and then obtain a representative of each alternative. Based on the representative payoff of each alternative, the final decision result is obtained. The decision making under measure-based granular uncertainty can not only simplify the decision-making process, but also effectively hold uncertain information. Although the decision making under measure-based granular uncertainty is a very efficient decision-making method based on unknown information, it cannot deal with the decision problem based on intuitionistic fuzzy environment. As the intuitionistic fuzzy environment is more general, there is an urgent need to put forward the decision making under measure-based uncertainty with intuitionistic fuzzy sets. However, what is the decision making under measure-based granular uncertainty with intuitionistic fuzzy sets is still an open issue to be addressed.

This paper proposes the decision making under measurebased granular uncertainty with intuitionistic fuzzy sets, which can apply the decision making under measurebased granular uncertainty to solve the problems under intuitionistic fuzzy environment, with the aid of Choquet integral, representative payoffs and measure. The flow of the decision making under measure-based granular uncertainty with intuitionistic fuzzy sets in this article requires the decision makers to determine how many candidate alternatives there are and the space. The granulation of the space should be determined. Moreover, relying on the actual problem, the payoffs of each alternative are given. The corresponding intuitionistic fuzzy sets of pasyoffs are also obtained. Membership degrees and non-membership degrees in intuitionistic fuzzy sets are integrated to obtain the integrated value. Then, the measure corresponding to the granulation will be obtained. The payoff bags of the problem based on the integrated value and granulation will be calculated. Hence, the corresponding representative payoff for each payoff bag can be obtained. According to the representative payoff of each payoff bag, get the representative payoff of each alternative. Based on the representative payoff of each alternative, the final decision result is obtained. In order to extend decision making under measure-based granular uncertainty with intuitionistic fuzzy sets to intuitionistic fuzzy environment, this model uses intuitionistic fuzzy set to represent payoffs of alternatives. If the intuitionistic fuzzy set is not used to express the payoffs of the alternatives, then decision making under measure-based granular uncertainty with intuitionistic fuzzy sets will degenerate into the decision making under measure-based granular uncertainty. Obviously, from the previous discussion, we know that the proposed model in this paper can be applied to the intuitionistic fuzzy environment, but the decision making under measure-based granular uncertainty cannot. This is the key difference between the model in this paper and his method is that the proposed model in this paper extends the decision making under measure-based granular uncertainty to the intuitionistic fuzzy environment, which is also the 
advantage of the proposed model in this paper. It means that the proposed model can handle the situation what the decision making under measure-based granular uncertainty can not.

The rest of the paper is structured as follows. Section 2 introduces the preliminary. Section 3 presents the decision making under measure-based granular uncertainty with intuitionistic fuzzy sets. Section 4 illustrates the flexibility and accuracy of the decision making under measure-based granular uncertainty with intuitionistic fuzzy sets. Section 5 summarizes the whole paper.

\section{Preliminaries}

Nowadays, there are a lot of uncertain issues [23-26]. The real world is very complicated [27-29]. The unknown is everywhere, just like no one knows why there will be an outbreak of COVID-19 in early 2020, no one knows when the next earthquake will come, no one knows when the next tsunami will come. To deal with these issues, many models and theories has been proposed and plenty of problems has been solved, such as decision making [30], resilience analysis [31], probability transform [32], pattern classification [33].

In this section, representative payoffs [22], intuitionistic fuzzy sets [34], measure [35] are briefly introduced.

\subsection{Measure}

Given a space $X$, the definition of measure is defined as follows:

Definition 1 (Measure) [36] Assume $\mu$ on $X$ is a mapping $\mu: X \rightarrow[0,1]$ and satisfies the follow conditions:

$\mu(X)=1$

$\mu(\emptyset)=0$

$\mu(A) \leq \mu(B)$ if $A \subseteq B$

Where, $A$ and $B$ are subsets of $X$.

Hence, $\mu$ is a measure.

The fuzzy measure is an effective tool to represent uncertainties, which can be combined with many other algorithms and models.

\subsection{Intuitionistic fuzzy sets}

There are many models to deal with uncertain information [37]. Intuitionistic fuzzy sets is an efficiency tool to represent uncertainty $[38,39]$. Assume $\Theta=\left\{z_{1}, z_{2}, \ldots, z_{n}\right\}$ is a frame of discernment and the power set of $\Theta$ is $2^{\Theta}=$ $\left\{\emptyset,\left\{z_{1}\right\}, \ldots,\left\{z_{n}\right\},\left\{z_{1}, z_{2}\right\}, \ldots,\left\{z_{1}, z_{2}, \ldots, z_{i}\right\}, \ldots, \Theta\right\}$.

The definition of an intuitionistic fuzzy set on $\Theta$ is as follows:

Definition 2 (Intuitionistic Fuzzy Sets) [34]

$B=\left\{\left\langle z_{i}, \mu_{B}\left(z_{i}\right), v_{B}\left(z_{i}\right) \mid z_{i} \in \Theta\right\rangle\right\}$

Where, $\mu_{B}\left(z_{i}\right)$ and $v_{B}\left(z_{i}\right): 2^{\Theta} \rightarrow[0,1]$ are membership degree and non-membership degree respectively, for any element of $2^{\Theta}, B$.

Intuitionistic fuzzy sets is the most famous extension of fuzzy sets. It is an expression of the real world [40]. So far, the research on this model has been very popular and mainstream at home and abroad [41].

If $A$ is an orthopair fuzzy set [42] and $q$ is the minimal value such that all $\left(A^{+}(z)^{q}+A^{-}(z)^{q}\right) \leq 1$, then $A$ is called $q$-rung orthopair fuzzy $\operatorname{set}(q$-ROFS). The definition of a mate interval value fuzzy set $D$ such that $D_{L}(z)=$ $A^{+}(z)$ and $D_{U}(z)=\left(1-\left(A^{-}(z)\right)^{q}\right)^{1 / q}$. Given a $q$-ROFS $A(z)=\left\langle A^{+}(z), A^{-}(z)\right\rangle$, Yager et al. [43] has proposed the following approach to obtain a possible membership grade of standard fuzzy subsets:

$V\left(z_{i}\right)=\left((1-\lambda)\left(D_{L}\left(z_{i}\right)\right)^{q}+\lambda\left(D_{U}\left(z_{i}\right)\right)^{q}\right)^{1 / q}$

Where, $\lambda \in[0,1]$.

\subsection{Representative payoffs}

Given a space $X$ with granular sets $F$. Meanwhile, $\lambda$ is a measure based on $F$. The definition of an representative payoffs of alternative $A_{k}$ is as follows:

Definition 3 (Representative payoffs) [22]

$\operatorname{Rep}\left(A_{k}\right)=\operatorname{Choq}_{\lambda}\left(\operatorname{Rep}\left(B_{k j}\right)\right.$ for $j=1$ to $\left.q\right)$

Where, $B_{k j}=\left\langle b_{k j r}\right.$ for $r=1$ to $\left.n_{j}\right\rangle$ with each $b_{k j r}=$ $a_{k i}$ for $x_{i} \in F_{j} . n_{j}$ is the number of payoffs in $B_{k j}$.

We use ordered weighted averaging operator to obtain $\operatorname{Rep}\left(A_{k}\right)$ by integrating $\operatorname{Rep}\left(B_{k j}\right)$ for $j=1$ to $q$. So in fact, other ordered weighted averaging methods clustering operator can be used to replace the Choquet integral in this definition.

\section{The proposed method}

Given a space $X$ with granular sets $F . \lambda$ is a measure based on $F$. Alternatives $A_{k}$ be represented as $A_{k}=$ $\left\{\left\langle a_{k r}, \mu\left(a_{k r}\right), v\left(a_{k r}\right)\right\rangle, r=1\right.$ to $\left.n_{k}\right\}$. The definition of an representative payoffs of alternative $A_{k}$ is as follows: 
Definition 4 (Decision making under measure-based granular uncertainty with intuitionistic fuzzy sets)

$$
\operatorname{Rep}\left(A_{k}\right)=\operatorname{Choq}_{\lambda}\left(\operatorname{Rep}\left(B_{k j}\right) \text { for } j=1 \text { to } q\right)
$$

Where, $B_{k j}=\left\langle b_{k j r}\right.$ for $r=1$ to $\left.n_{j}\right\rangle$ with each $b_{k j r}$ is the grade of $a_{k i}$ for $x_{i} \in F_{j}$ obtained by (5). $n_{j}$ is the number of payoffs in $B_{k j}$.

With $\rho$ is an index function such that $\rho(i)$ is the index of granular set with the $i$ th largest value for $\operatorname{Rep}\left(B_{k j}\right)$, then

$$
\begin{aligned}
& \operatorname{Choq}_{\lambda}\left(\operatorname{Rep}\left(B_{k j}\right) \text { for } j=1 \text { to } q\right)=\sum_{i=1}^{q}\left(\lambda\left(H_{i}\right)\right. \\
& \left.-\lambda\left(H_{i-1}\right)\right) \operatorname{Rep}\left(B_{k \rho(i)}\right)
\end{aligned}
$$

Where $H_{i}=\left\{F_{\rho(1)}, F_{\rho(2)}, \ldots, F_{\rho(i)}\right\}$, the collection of the $i$ granular sets with the $i$ th largest values for $\operatorname{Rep}\left(B_{k \rho(i)}\right)$.

In particular, the representative value of $B_{k j}$ can be represented as follows:

$$
\begin{aligned}
& \operatorname{Rep}\left(B_{k j}\right)=\min _{r=1 \text { to } n_{j}}\left[b_{k j r}\right] \\
& \operatorname{Rep}\left(B_{k j}\right)=\max _{r=1 \text { to } n_{j}}\left[b_{k j r}\right] \\
& \operatorname{Rep}\left(B_{k j}\right)=\frac{1}{n_{j}} \sum_{r=1}^{n_{j}} b_{k j r} \\
& \operatorname{Rep}\left(B_{k j}\right)=\operatorname{Middle}\left(b_{k j r}\right)
\end{aligned}
$$

Theorem 1 Assume $\lambda$ is a probability measure such that $\lambda\left(\left\{F_{j}\right\}\right)=p_{j}$. Then $\operatorname{Rep}\left(A_{k}\right)=\sum_{i=1}^{q} p_{i} \operatorname{Rep}\left(B_{k i}\right)$.

Proof Since $\lambda$ is a probability measure, for any subset $D$ of $F$, we have $\lambda(D)=\sum_{F_{j} \in D} p_{j}$ such that $\lambda\left(H_{i}\right)-$ $\lambda\left(H_{i-1}\right)=p_{\rho(i)}$.

Relying on the equations of (8), we have equations as follows:

$$
\begin{aligned}
& \operatorname{Choq}_{\lambda}\left(\operatorname{Rep}\left(B_{k j}\right) \text { for } j=1 \text { to } q\right) \\
= & \sum_{i=1}^{q}\left(\lambda\left(H_{i}\right)-\lambda\left(H_{i-1}\right)\right) \operatorname{Rep}\left(B_{k \rho(i)}\right) \\
= & \sum_{i=1}^{q} p_{\rho(i)} \operatorname{Rep}\left(B_{k \rho(i)}\right) \\
= & \sum_{i=1}^{q} p_{i} \operatorname{Rep}\left(B_{k i}\right)
\end{aligned}
$$

Hence, we obtain

$\operatorname{Rep}\left(A_{k}\right)=\sum_{i=1}^{q} p_{i} \operatorname{Rep}\left(B_{k i}\right)$

Theorem 2 Assume $\lambda$ is a cardinality-based measure with parameters $1=a_{q} \geq \ldots \geq a_{1} \geq a_{0}=0$. Then $\operatorname{Rep}\left(A_{k}\right)=$ $\sum_{i=1}^{q}\left(a_{i}-a_{i-1}\right) \operatorname{Rep}\left(B_{k \rho(i)}\right)$.
Proof Since $\lambda$ is a cardinality-based measure, so, for any subset $B$ of $F$, we have $\lambda(B)=a_{|B|}$ so that $\lambda\left(H_{i}\right)-$ $\lambda\left(H_{i-1}\right)=a_{i}-a_{i-1}$.

Relying on the equations of (8), we have equations as follows:

$$
\begin{aligned}
& \operatorname{Choq}_{\lambda}\left(\operatorname{Rep}\left(B_{k j}\right) \text { for } j=1 \text { to } q\right) \\
= & \sum_{i=1}^{q}\left(\lambda\left(H_{i}\right)-\lambda\left(H_{i-1}\right)\right) \operatorname{Rep}\left(B_{k \rho(i)}\right) \\
= & \sum_{i=1}^{q}\left(a_{i}-a_{i-1}\right) \operatorname{Rep}\left(B_{k \rho(i)}\right)
\end{aligned}
$$

Hence, we obtain

$\operatorname{Rep}\left(A_{k}\right)=\sum_{i=1}^{q}\left(a_{i}-a_{i-1}\right) \operatorname{Rep}\left(B_{k \rho(i)}\right)$

Theorem 3 Assume $\lambda$ is a maxitive measure with $\lambda\left(\left\{F_{j}\right\}\right)$ $=\pi_{j}$. Then $\operatorname{Rep}\left(A_{k}\right)=\sum_{i=1}^{q}\left(0 \vee\left(\pi_{\rho(i)}-\max _{d=1}\right.\right.$ to $i-1$ $\left.\left.\left[\pi_{\rho(d)}\right]\right)\right) \operatorname{Rep}\left(B_{k \rho(i)}\right)$.

Proof Since $\lambda$ is a maxitive measure with $\lambda\left(\left\{F_{j}\right\}\right)=\pi_{j}$, so, for any subset $D$ of $F, \lambda(D)=\max _{F_{j} \in D}\left[\pi_{j}\right]$ so that $\lambda\left(H_{i}\right)-\lambda\left(H_{i-1}\right)=\max _{F_{j} \in H_{i}}\left[\pi_{j}\right]-\max _{F_{j} \in H_{i-1}}\left[\pi_{j}\right]$.

Relying on the equations of (8), we have equations as follows:

$$
\begin{aligned}
& \operatorname{Choq}_{\lambda}\left(\operatorname{Rep}\left(B_{k j}\right) \text { for } j=1 \text { to } q\right) \\
= & \sum_{i=1}^{q}\left(\lambda\left(H_{i}\right)-\lambda\left(H_{i-1}\right)\right) \operatorname{Rep}\left(B_{k \rho(i)}\right) \\
= & \sum_{i=1}^{q}\left(\max _{F_{j} \in H_{i}}\left[\pi_{j}\right]-\max _{\left.F_{j} \in H_{i-1}\left[\pi_{j}\right]\right) \operatorname{Rep}\left(B_{k \rho(i)}\right)}\right. \\
= & \sum_{i=1}^{q}\left(0 \vee\left(\pi_{\rho(i)}-\max _{d=1 \text { to } i-1}\left[\pi_{\rho(d)}\right]\right)\right) \operatorname{Rep}\left(B_{k \rho(i)}\right)
\end{aligned}
$$

Hence, we obtain

$$
\operatorname{Rep}\left(A_{k}\right)=\sum_{i=1}^{q}\left(0 \vee\left(\pi_{\rho(i)}-\max _{d=1 \text { to } i-1}\left[\pi_{\rho(d)}\right]\right)\right) \operatorname{Rep}\left(B_{k \rho(i)}\right)
$$

The flow of decision making under measure-based granular uncertainty with intuitionistic fuzzy sets can be shown as Fig. 1.

Based on the Fig. 1, the steps of the proposed model in this paper are as follows:

step 1 Determine how many candidate alternatives there are and the space.

step 2 The granulation of the space should be determined.

step 3 Relying on the actual problem, the possible payoffs of each alternative are given.

step 4 Obtaining the corresponding intuitionistic fuzzy sets of possible payoffs. 


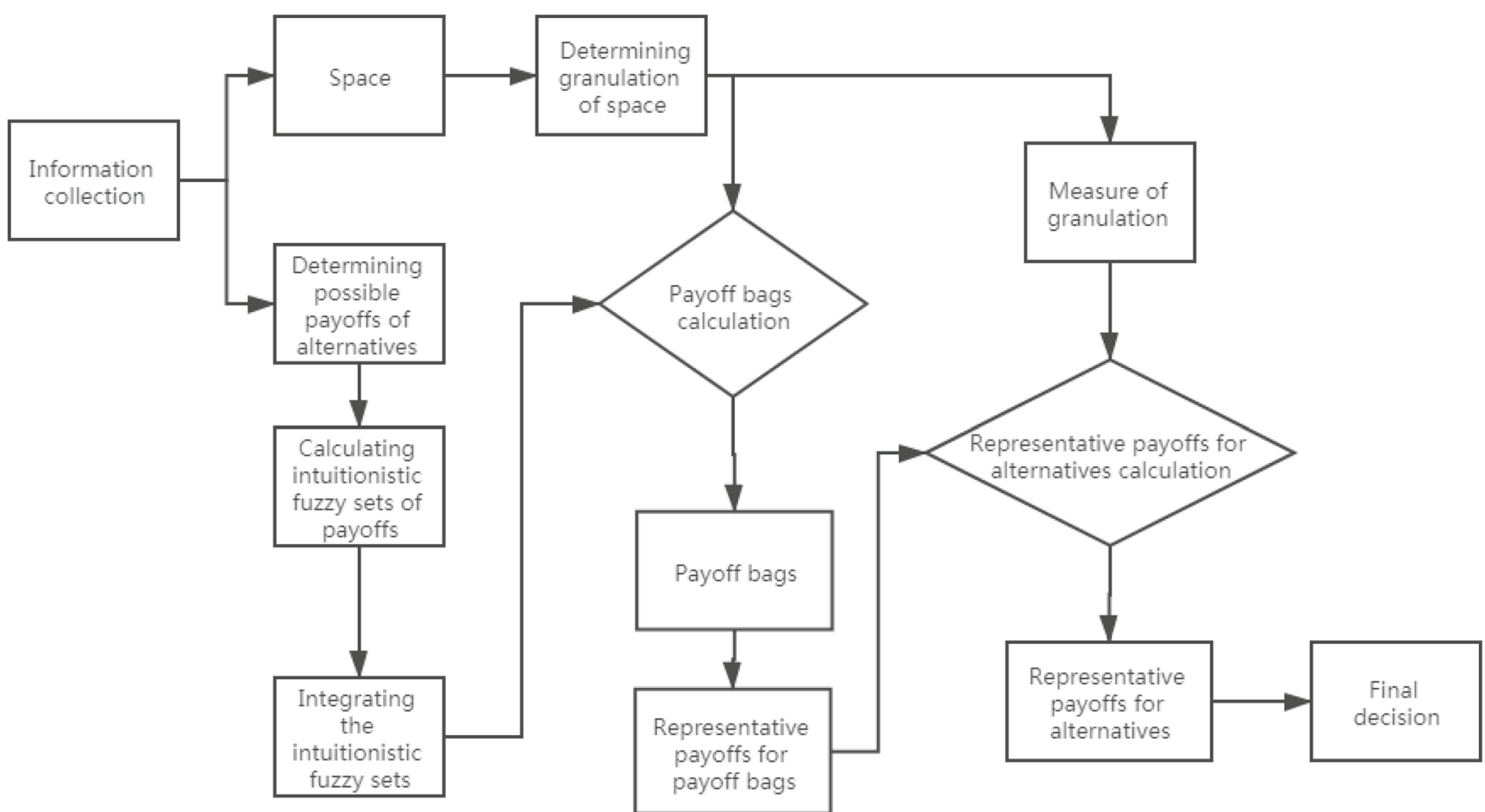

Fig. 1 The flow of decision making under measure-based granular uncertainty with intuitionistic fuzzy sets

step 5 Integrating these membership degrees and nonmembership degrees in intuitionistic fuzzy sets to obtain the integrated values by (5).

step 6 Obtaining the measure corresponding to the granulation.

step 7 Calculating the payoff bags of the problem based on the integrated values and granulation.

step 8 Obtaining the corresponding representative payoff for each payoff bag by (8).

step 9 Relying on the representative payoff of each payoff bag and measure, get the representative payoff of each alternative by (7).

step 10 Relying on the representative payoff of each alternative, the final decision result is obtained.

The decision making under measure-based granular uncertainty with intuitionistic fuzzy sets converts possible payoffs of the alternatives in issues into the form of intuitionistic fuzzy sets, which is a very flexible model. That is to say, the decision making under measure-based granular uncertainty with intuitionistic fuzzy sets extends decision making under measure-based granular uncertainty to the intuitionistic fuzzy environment, which is both a generalization and an innovation of decision making under measure-based granular uncertainty with intuitionistic fuzzy sets. Since the intuitionistic fuzzy environment represented by the intuitionistic fuzzy set is an environment more close to the real world, the decision making under measure-based granular uncertainty with intuitionistic fuzzy sets is more widely applied than decision making under measure-based granular uncertainty. If the intuitionistic fuzzy set is not used to express the payoffs of the alternatives, then decision making under measure-based granular uncertainty with intuitionistic fuzzy sets will degenerate into the decision making under measure-based granular uncertainty.

\section{Numerical examples}

Example 1 Assume $A_{1}$ and $A_{2}$ are two alternatives with a space $X=\left\{x_{1}, x_{2}, x_{3}, x_{4}\right\}$. The possible payoffs of two alternatives $A_{1}$ and $A_{2}$ are as follows:

$$
\begin{aligned}
& a_{11}=10, a_{12}=30, a_{13}=15, a_{14}=20 \\
& a_{21}=12, a_{22}=24, a_{23}=18, a_{24}=22
\end{aligned}
$$

The related intuitionistic fuzzy sets under two alternatives are as follows:

$$
\begin{aligned}
A_{1}= & \left\{\left\langle a_{11}, 0.3,0.5\right\rangle,\left\langle a_{12}, 0.5,0.2\right\rangle,\left\langle a_{13}, 0.7,0.1\right\rangle,\right. \\
& \left.\left\langle a_{14}, 0.2,0.2\right\rangle\right\} \\
A_{2}= & \left\{\left\langle a_{21}, 0.5,0.2\right\rangle,\left\langle a_{22}, 0.2,0.4\right\rangle,\left\langle a_{23}, 0.5,0.3\right\rangle,\right. \\
& \left.\left\langle a_{24}, 0.5,0.4\right\rangle\right\}
\end{aligned}
$$

Relying on the (5), the grades of $A_{1}$ and $A_{2}$ are as follows:

$$
\begin{aligned}
V\left(a_{11}\right)= & 0.4, V\left(a_{12}\right)=0.65, V\left(a_{13}\right)=0.8, \\
& V\left(a_{14}\right)=0.5 \\
V\left(a_{21}\right)= & 0.65, V\left(a_{22}\right)=0.4, V\left(a_{23}\right)=0.6, \\
& V\left(a_{24}\right)=0.55
\end{aligned}
$$


The granulation of $X, F=\left\{F_{1}, F_{2}, F_{3}\right\}$, is as follows:

$F_{1}=\left\{x_{1}, x_{2}\right\}, F_{2}=\left\{x_{2}, x_{3}\right\}, F_{3}=\left\{x_{1}, x_{3}, x_{4}\right\}$

The measure on $F, \lambda$, is as follows:

$\lambda(\emptyset)=0, \lambda\left(F_{1}\right)=0.2, \lambda\left(F_{2}\right)=0.3, \lambda\left(F_{3}\right)=0.3$

$\lambda\left(\left\{F_{1}, F_{2}\right\}\right)=0.4, \lambda\left(\left\{F_{1}, F_{3}\right\}\right)=0.4, \lambda\left(\left\{F_{2}, F_{3}\right\}\right)=0.5$,

$$
\lambda(F)=1
$$

The payoffs bags are as follows:

$B_{11}=\langle 0.4,0.65\rangle, B_{12}=\langle 0.65,0.8\rangle, B_{13}=\langle 0.4,0.8,0.5\rangle$

$B_{21}=\langle 0.65,0.4\rangle, B_{22}=\langle 0.4,0.6\rangle$,

$B_{23}=\langle 0.65,0.6,0.55\rangle$

Calculate the values of $\operatorname{Rep}\left(B_{k j}\right)$, as follows:

$\operatorname{Rep}\left(B_{11}\right)=0.525, \operatorname{Rep}\left(B_{12}\right)=0.725, \operatorname{Rep}\left(B_{13}\right)=0.567$

$\operatorname{Rep}\left(B_{21}\right)=0.525, \operatorname{Rep}\left(B_{22}\right)=0.5, \operatorname{Rep}\left(B_{23}\right)=0.6$

Hence, calculate $\operatorname{Rep}\left(A_{1}\right)$ and $\operatorname{Rep}\left(A_{2}\right)$ as follows:

$$
\begin{aligned}
\operatorname{Rep}\left(A_{1}\right) & =\operatorname{Choq}_{\lambda}\left(\operatorname{Rep}\left(B_{11}\right), \operatorname{Rep}\left(B_{12}\right), \operatorname{Rep}\left(B_{13}\right)\right) \\
& =0.3 \times 0.725+0.2 \times 0.567+0.5 \times 0.525 \\
& =0.5934 \\
\operatorname{Rep}\left(A_{2}\right) & =\operatorname{Choq}_{\lambda}\left(\operatorname{Rep}\left(B_{21}\right), \operatorname{Rep}\left(B_{22}\right), \operatorname{Rep}\left(B_{23}\right)\right) \\
& =0.3 \times 0.6+0.1 \times 0.525+0.6 \times 0.5=0.5325
\end{aligned}
$$

Since $\operatorname{Rep}\left(A_{1}\right)>\operatorname{Rep}\left(A_{2}\right)$, the $A_{1}$ is the best alternative.

Example 2 Recently, there was a robbery in a mall. If there are three suspects, and one of them is a criminal, it is impossible to tell which of the three is guilty according to the existing model. So we have invited four decision experts to use the proposed model in this paper to determine who is the real criminal at this time. Assume there are three suspects $A_{1}, A_{2}$ and $A_{3}$ with a space $X=\left\{x_{1}, x_{2}, x_{3}, x_{4}\right\}$ in a case. The possible payoffs of three alternatives are as follows:

$$
\begin{aligned}
& a_{11}=24, a_{12}=25, a_{13}=12, a_{14}=18 \\
& a_{21}=15, a_{22}=18, a_{23}=27, a_{24}=32 \\
& a_{31}=29, a_{32}=32, a_{33}=6, a_{34}=12
\end{aligned}
$$

The related intuitionistic fuzzy sets under three alternatives are as follows:

$$
\begin{aligned}
A_{1}= & \left\{\left\langle a_{11}, 0.2,0.5\right\rangle,\left\langle a_{12}, 0.4,0.3\right\rangle,\left\langle a_{13}, 0.8,0.1\right\rangle,\right. \\
& \left.\left\langle a_{14}, 0.3,0.3\right\rangle\right\} \\
A_{2}= & \left\{\left\langle a_{21}, 0.5,0.3\right\rangle,\left\langle a_{22}, 0.3,0.1\right\rangle,\left\langle a_{23}, 0.4,0.2\right\rangle,\right. \\
& \left.\left\langle a_{24}, 0.1,0.4\right\rangle\right\} \\
A_{3}= & \left\{\left\langle a_{31}, 0.1,0.6\right\rangle,\left\langle a_{32}, 0.6,0.4\right\rangle,\left\langle a_{33}, 0.3,0.3\right\rangle,\right. \\
& \left.\left\langle a_{34}, 0.2,0.5\right\rangle\right\}
\end{aligned}
$$

Relying on the (5), the grades of these suspects are as follows:

$$
\begin{aligned}
V\left(a_{11}\right)= & 0.35, V\left(a_{12}\right)=0.55, V\left(a_{13}\right)=0.85 \\
& V\left(a_{14}\right)=0.5 \\
V\left(a_{21}\right)= & 0.6, V\left(a_{22}\right)=0.6, V\left(a_{23}\right)=0.6, V\left(a_{24}\right)=0.35 \\
V\left(a_{31}\right)= & 0.25, V\left(a_{32}\right)=0.6, V\left(a_{33}\right)=0.5, \\
& V\left(a_{34}\right)=0.35
\end{aligned}
$$

The granulation of $X, F=\left\{F_{1}, F_{2}, F_{3}\right\}$, is as follows:

$F_{1}=\left\{x_{1}, x_{3}\right\}, F_{2}=\left\{x_{2}, x_{4}\right\}, F_{3}=\left\{x_{1}, x_{2}, x_{3}\right\}$

The measure on $F, \lambda$, is as follows:

$\lambda(\emptyset)=0, \lambda\left(F_{1}\right)=0.4, \lambda\left(F_{2}\right)=0.2, \lambda\left(F_{3}\right)=0.5$

$\lambda\left(\left\{F_{1}, F_{2}\right\}\right)=0.6, \lambda\left(\left\{F_{1}, F_{3}\right\}\right)=0.8, \lambda\left(\left\{F_{2}, F_{3}\right\}\right)$

$=0.7, \lambda(F)=1$

The payoffs bags are as follows:

$$
\begin{aligned}
B_{11}=\langle 0.35,0.85\rangle, B_{12}=\langle 0.55,0.5\rangle, & \\
& B_{13}=\langle 0.35,0.55,0.85\rangle \\
B_{21}= & \langle 0.6,0.6\rangle, B_{22}=\langle 0.6,0.35\rangle, B_{23}=\langle 0.6,0.6,0.6\rangle \\
B_{31}= & \langle 0.25,0.5\rangle, B_{32}=\langle 0.6,0.35\rangle, B_{33}=\langle 0.25,0.6,0.5\rangle
\end{aligned}
$$

Calculate the values of $\operatorname{Rep}\left(B_{k j}\right)$, as follows:

$$
\begin{aligned}
& \operatorname{Rep}\left(B_{11}\right)=0.6, \operatorname{Rep}\left(B_{12}\right)=0.525, \operatorname{Rep}\left(B_{13}\right)=0.583 \\
& \operatorname{Rep}\left(B_{21}\right)=0.6, \operatorname{Rep}\left(B_{22}\right)=0.475, \operatorname{Rep}\left(B_{23}\right)=0.6 \\
& \operatorname{Rep}\left(B_{31}\right)=0.375, \operatorname{Rep}\left(B_{32}\right)=0.475, \operatorname{Rep}\left(B_{33}\right)=0.675
\end{aligned}
$$

Hence, calculate $\operatorname{Rep}\left(A_{1}\right), \operatorname{Rep}\left(A_{2}\right)$ and $\operatorname{Rep}\left(A_{3}\right)$ as follows:

$$
\begin{aligned}
\operatorname{Rep}\left(A_{1}\right) & =C_{\operatorname{Choq}}\left(\operatorname{Rep}\left(B_{11}\right), \operatorname{Rep}\left(B_{12}\right), \operatorname{Rep}\left(B_{13}\right)\right) \\
& =0.4 \times 0.6+0.4 \times 0.583+0.2 \times 0.525 \\
& =0.5782 \\
\operatorname{Rep}\left(A_{2}\right) & =C h o q_{\lambda}\left(\operatorname{Rep}\left(B_{21}\right), \operatorname{Rep}\left(B_{22}\right), \operatorname{Rep}\left(B_{23}\right)\right) \\
& =0.4 \times 0.6+0.4 \times 0.6+0.2 \times 0.475=0.575 \\
\operatorname{Rep}\left(A_{3}\right) & =C h o q_{\lambda}\left(\operatorname{Rep}\left(B_{31}\right), \operatorname{Rep}\left(B_{32}\right), \operatorname{Rep}\left(B_{33}\right)\right) \\
& =0.5 \times 0.675+0.2 \times 0.475+0.3 \times 0.375 \\
& =0.545
\end{aligned}
$$

Since $\operatorname{Rep}\left(A_{1}\right)>\operatorname{Rep}\left(A_{2}\right)>\operatorname{Rep}\left(A_{3}\right)$, the $A_{1}$ is the most likely culprit.

\section{Application}

In order to compare the advantages and disadvantages of decision making under measure-based granular uncertainty with intuitionistic fuzzy sets with decision making under measure-based granular uncertainty, the following practical application of applied intelligence is given in this paper. Since the beginning of 2020, COVID-19 has been ravaging the human earth, and human life has been severely affected. In order to treat COVID-19, suppose that two drugs have been developed, and each drug is scored by four experts. The scoring of four experts constitutes the space for this question. We are now evaluating both drugs using decision making under measure-based granular uncertainty with intuitionistic fuzzy sets and decision making under measure-based granular uncertainty, respectively. First, we evaluated the two drugs using the decision making under measure-based granular uncertainty, and the evaluation process is as follows: 
According to the previous explanation, $A_{1}$ and $A_{2}$ are two drugs with a space $X=\left\{x_{1}, x_{2}, x_{3}, x_{4}\right\}$. The possible payoffs of two drugs $A_{1}$ and $A_{2}$ are as follows:

$a_{11}=15, a_{12}=30, a_{13}=20, a_{14}=10$

$a_{21}=10, a_{22}=24, a_{23}=15, a_{24}=20$.

The granulation of $X, F=\left\{F_{1}, F_{2}, F_{3}\right\}$, is as follows:

$F_{1}=\left\{x_{1}, x_{2}\right\}, F_{2}=\left\{x_{2}, x_{3}\right\}, F_{3}=\left\{x_{1}, x_{3}, x_{4}\right\}$

The measure on $F, \lambda$, is as follows:

$$
\begin{aligned}
& \lambda(\emptyset)=0, \lambda\left(F_{1}\right)=0.2, \lambda\left(F_{2}\right)=0.3, \lambda\left(F_{3}\right)=0.3 \\
& \begin{aligned}
\lambda\left(\left\{F_{1}, F_{2}\right\}\right) & =0.4, \lambda\left(\left\{F_{1}, F_{3}\right\}\right)=0.4, \lambda\left(\left\{F_{2}, F_{3}\right\}\right) \\
& =0.5, \lambda(F)=1
\end{aligned}
\end{aligned}
$$

The payoffs bags are as follows:

$B_{11}=\langle 15,30\rangle, B_{12}=\langle 30,20\rangle, B_{13}=\langle 15,20,10\rangle$

$B_{21}=\langle 10,24\rangle, B_{22}=\langle 24,15\rangle, B_{23}=\langle 10,15,20\rangle$

Calculate the values of $\operatorname{Rep}\left(B_{k j}\right)$, as follows:

$\operatorname{Rep}\left(B_{11}\right)=22.5, \operatorname{Rep}\left(B_{12}\right)=25, \operatorname{Rep}\left(B_{13}\right)=15$

$\operatorname{Rep}\left(B_{21}\right)=17, \operatorname{Rep}\left(B_{22}\right)=19.5, \operatorname{Rep}\left(B_{23}\right)=15$

Hence, calculate $\operatorname{Rep}\left(A_{1}\right)$ and $\operatorname{Rep}\left(A_{2}\right)$ as follows:

$$
\begin{aligned}
\operatorname{Rep}\left(A_{1}\right) & =\operatorname{Choq}_{\lambda}\left(\operatorname{Rep}\left(B_{11}\right), \operatorname{Rep}\left(B_{12}\right), \operatorname{Rep}\left(B_{13}\right)\right) \\
& =0.3 \times 25+0.1 \times 22.5+0.6 \times 15=18.75 \\
\operatorname{Rep}\left(A_{2}\right) & =\operatorname{Choq}_{\lambda}\left(\operatorname{Rep}\left(B_{21}\right), \operatorname{Rep}\left(B_{22}\right), \operatorname{Rep}\left(B_{23}\right)\right) \\
& =0.3 \times 19.5+0.1 \times 17+0.6 \times 15=16.55
\end{aligned}
$$

Since $\operatorname{Rep}\left(A_{1}\right)>\operatorname{Rep}\left(A_{2}\right)$, the $A_{1}$ is a better drug than $A_{1}$.

Then, we evaluated the two drugs using the decision making under measure-based granular uncertainty with intuitionistic fuzzy sets, and the evaluation process is as follows:

According to the previous explanation, we know that $A_{1}$ and $A_{2}$ are two drug with a space $X=\left\{x_{1}, x_{2}, x_{3}, x_{4}\right\}$. The possible payoffs of two drugs $A_{1}$ and $A_{2}$ are as follows:

$$
\begin{aligned}
& a_{11}=15, a_{12}=30, a_{13}=20, a_{14}=10 \\
& a_{21}=10, a_{22}=24, a_{23}=15, a_{24}=20
\end{aligned}
$$

The related intuitionistic fuzzy sets under two alternatives are as follows:

$$
\begin{aligned}
A_{1}= & \left\{\left\langle a_{11}, 0.2,0.3\right\rangle,\left\langle a_{12}, 0.3,0.4\right\rangle,\left\langle a_{13}, 0.6,0.1\right\rangle,\right. \\
& \left.\left\langle a_{14}, 0.2,0.2\right\rangle\right\} \\
A_{2}= & \left\{\left\langle a_{21}, 0.5,0.3\right\rangle,\left\langle a_{22}, 0.5,0.4\right\rangle,\left\langle a_{23}, 0.7,0.3\right\rangle,\right. \\
& \left.\left\langle a_{24}, 0.3,0.3\right\rangle\right\}
\end{aligned}
$$

Relying on the (5), the grades of $A_{1}$ and $A_{2}$ are as follows:

$$
\begin{aligned}
V\left(a_{11}\right)= & 0.25, V\left(a_{12}\right)=0.35, V\left(a_{13}\right)=0.35 \\
& V\left(a_{14}\right)=0.2 \\
V\left(a_{21}\right)= & 0.4, V\left(a_{22}\right)=0.45, V\left(a_{23}\right)=0.5, V\left(a_{24}\right)=0.3
\end{aligned}
$$

The granulation of $X, F=\left\{F_{1}, F_{2}, F_{3}\right\}$, is as follows:

$$
F_{1}=\left\{x_{1}, x_{2}\right\}, F_{2}=\left\{x_{2}, x_{3}\right\}, F_{3}=\left\{x_{1}, x_{3}, x_{4}\right\}
$$

The measure on $F, \lambda$, is as follows:

$$
\begin{aligned}
& \lambda(\emptyset)=0, \lambda\left(F_{1}\right)=0.2, \lambda\left(F_{2}\right)=0.3, \lambda\left(F_{3}\right)=0.3 \\
& \begin{aligned}
\lambda\left(\left\{F_{1}, F_{2}\right\}\right) & =0.4, \lambda\left(\left\{F_{1}, F_{3}\right\}\right)=0.4, \lambda\left(\left\{F_{2}, F_{3}\right\}\right) \\
& =0.5, \lambda(F)=1
\end{aligned}
\end{aligned}
$$

The payoffs bags are as follows:

$$
\begin{aligned}
& B_{11}=\langle 0.25,0.35\rangle, B_{12}=\langle 0.35,0.35\rangle, \\
& B_{13}=\langle 0.25,0.35,0.2\rangle \\
& B_{21}=\langle 0.4,0.45\rangle, B_{22}=\langle 0.45,0.5\rangle, \\
& B_{23}=\langle 0.4,0.5,0.3\rangle
\end{aligned}
$$

Calculate the values of $\operatorname{Rep}\left(B_{k j}\right)$, as follows:

$$
\begin{aligned}
& \operatorname{Rep}\left(B_{11}\right)=0.3, \operatorname{Rep}\left(B_{12}\right)=0.35, \operatorname{Rep}\left(B_{13}\right)=0.266 \\
& \operatorname{Rep}\left(B_{21}\right)=0.425, \operatorname{Rep}\left(B_{22}\right)=0.475, \operatorname{Rep}\left(B_{23}\right)=0.4
\end{aligned}
$$

Hence, calculate $\operatorname{Rep}\left(A_{1}\right)$ and $\operatorname{Rep}\left(A_{2}\right)$ as follows:

$$
\begin{aligned}
\operatorname{Rep}\left(A_{1}\right) & =\operatorname{Choq}_{\lambda}\left(\operatorname{Rep}\left(B_{11}\right), \operatorname{Rep}\left(B_{12}\right), \operatorname{Rep}\left(B_{13}\right)\right) \\
& =0.3 \times 0.35+0.1 \times 0.3+0.6 \times 0.266=0.2946 \\
\operatorname{Rep}\left(A_{2}\right) & =\operatorname{Choq}_{\lambda}\left(\operatorname{Rep}\left(B_{21}\right), \operatorname{Rep}\left(B_{22}\right), \operatorname{Rep}\left(B_{23}\right)\right) \\
& =0.3 \times 0.475+0.1 \times 0.425+0.6 \times 0.4=0.425
\end{aligned}
$$

Since $\operatorname{Rep}\left(A_{2}\right)>\operatorname{Rep}\left(A_{1}\right)$, the $A_{2}$ is a better drug than $A_{1}$.

Above all, we can see that the experimental result obtained by the decision making under measure-based granular uncertainty with intuitionistic fuzzy sets is different from that obtained by the decision making under measure-based granular uncertainty. At the same time, the decision making under measure-based granular uncertainty with intuitionistic fuzzy sets can accurately predict the second drug than the first one is more suitable for treatment of COVID-19, which is consistent with the effect predicted by experts. This not only indicates that the decision making under measurebased granular uncertainty with intuitionistic fuzzy sets is an extension of the decision making under measure-based granular uncertainty in the intuitionistic fuzzy environment, but also indicates that the decision making under measurebased granular uncertainty with intuitionistic fuzzy sets is more effective than the decision making under measurebased granular uncertainty in dealing with issues of decision making.

\section{Discussion}

In real world, there are many uncertainties [44, 45]. Meanwhile, many scholars proposed a lot of effective methods and theories to address these problems [46, 47]. The intuitionistic fuzzy sets is an extent of classical fuzzy sets $[48,49]$. Intuitionistic fuzzy set is an effective means to reflect the real world [50]. It divides the information contained in an unknown event into membership degree, nonmembership degree and hesitation degree, which are also the original representation methods of the real world [51]. For example, if a person has a cough or fever, the doctor 
will determine whether the person has COVID-19 or not. At this point, the membership degree is the probability that the person will get COVID-19. Non-membership degree is the probability that the person does not have COVID-19. Hesitancy degree is the probability that the doctor can't tell if the person has COVID-19. Yager proposed the decision making under measure-based granular uncertainty [22], which has promising aspects [52]. However, the decision making under measure-based granular uncertainty can not hold the decision making issues under intuitionistic fuzzy environment. This paper proposes the decision making under measure-based granular uncertainty with intuitionistic fuzzy sets, which is an extent of the decision making under measure-based granular uncertainty under the intuitionistic fuzzy environment. The proposed model is based on the intuitionistic fuzzy sets, which is an effective tool to represent uncertainties. On the other hand, if a system is under intuitionistic fuzzy environment, then the proposed model can deal with these issues of this system. The proposed model process in this article requires the decision maker to determine how many alternatives there are and the granulation of space. Then, the practical problems are analyzed and the payoffs of each alternative are given. Based on the payoffs, the corresponding intuitionistic fuzzy sets are also obtained. The integrated values are obtained by integrating the membership degrees and non-membership degrees of these intuitionistic fuzzy sets. Relying on the granulation, the measure corresponding to granulation is given. Then, the payoff bags are obtained based on these integrated values and granulation of space. Representative payoffs for these payoff bags can be obtained. Hence, based on the representation payoffs, the representative payoffs of these alternatives respectively are obtained. Finally, the final decision making result is obtained according to the representative payoffs of these alternatives. We can notice that the fundamental difference between the proposed model in this paper and the decision making under measure-based granular uncertainty is that the proposed model in this paper will convert the possible payoffs of each candidate into the corresponding intuitionistic fuzzy set, and integrate the membership degrees and non-membership degrees of the intuitionistic fuzzy sets by relevant methods. The integrated values will be aggregated by OWA operator, such as Choquet integral. In fact, Choquet integrals can be replaced by other OWA operators. This shows that the model in this paper can adapt to the real world, because the intuitionistic fuzzy sets can perfectly reflect the unknown information in the real world. So extending the decision making under measure-based granular uncertainty to an intuitionistic fuzzy environment also means that the proposed model in this paper is more flexible than the decision making under measure-based granular uncertainty. Numerical examples are applied to verify the validity of the decision making under measure-based granular uncertainty with intuitionistic fuzzy sets. The experimental results demonstrate that the decision making under measure-based granular uncertainty with intuitionistic fuzzy sets can represent the objects successfully and make decision effectively. In addition, a practical application of applied intelligence is used to compare the performance between the proposed model and the decision making under measure-based granular uncertainty. The experimental results show that the proposed model can solve some decision problems that the decision making under measure-based granular uncertainty cannot solve. In the future, we will extend the application of the proposed model to more wide scope, such as Pythagorean fuzzy environment, orthopair fuzzy environment.

\section{Conclusion}

This paper proposes the decision making under measurebased granular uncertainty with intuitionistic fuzzy sets, which can handle the issues under intuitionistic fuzzy environment. It means that the proposed model is an extend of the decision making under measure-based granular uncertainty. When the environment of the proposed model degenerate into the normal environment, the proposed model will degenerate into the decision making under measure-based granular uncertainty. The proposed model can represent the alternatives as possible payoffs. Then, the related intuitionistic fuzzy sets of possible payoffs can be obtained. The integrated values are obtained by integrating the membership degrees and non-membership degrees of these intuitionistic fuzzy sets. Then, the granulation of space should be given. Then, the payoff bags are obtained based on these integrated values and granulation of space. Representative payoffs for these payoff bags can be obtained. Hence, based on the representation payoffs, the representative payoffs of these alternatives respectively are obtained. Finally, the final decision making result is obtained according to the representative payoffs of these alternatives. This proposed model can combine the advantages of the decision making under measurebased granular uncertainty and intuitionistic fuzzy sets and make an accurate estimation of the alternatives. Numerical examples are applied to verify the validity of the decision making under measure-based granular uncertainty with intuitionistic fuzzy sets. The experimental results demonstrate that the decision making under measurebased granular uncertainty with intuitionistic fuzzy sets can represent the objects successfully and make decision effectively. In addition, a practical application of applied intelligence is used to compare the performance between the proposed model and the decision making under measurebased granular uncertainty. The experimental results show 
that the proposed model can solve some decision problems that the decision making under measure-based granular uncertainty cannot solve.

Funding The work is partially supported by National Natural Science Foundation of China (Grant No. 61973332).

\section{Declarations}

Ethics approval and consent to participate This article does not contain any studies with human participants or animals performed by any of the authors.

Conflict of Interests All the authors certify that there is no conflict of interest with any individual or organization for the present work.

\section{References}

1. Dzitac I, Filip FG, Manolescu M-J (2017) Fuzzy logic is not fuzzy: World-renowned computer scientist lotfi a. zadeh. Int J Comput Commun Control 12(6):748-789

2. Deng Y (2020) Information volume of mass function. Int $\mathbf{J}$ Comput Commun Control 15(6):3983

3. Pan Y, Zhang L, Li Z, Ding L (2019) Improved fuzzy bayesian network-based risk analysis with interval-valued fuzzy sets and d-s evidence theory. IEEE Trans Fuzzy Syst, 1-1

4. Gao X, Deng Y (2020) The pseudo-pascal triangle of maximum deng entropy. Int J Comput Commun Control 15(1):1006

5. Yang X, Li T, Liu D, Fujita H (2019) A temporal-spatial composite sequential approach of three-way granular computing. Inform Sci 486:02

6. Athira TM, John J, Garg H (2019) A novel entropy measure of pythagorean fuzzy soft sets

7. Wan Mohd WR, Abdullah L, Yusoff B, Taib CMIC, Merigo JM (2019) An integrated mcdm model based on pythagorean fuzzy sets for green supplier development program. Malaysian J Math Sci 13:23-37

8. Jiang W, Cao Y, Deng X (2019) A Novel Z-network Model Based on Bayesian Network and Z-number. IEEE Transactions on Fuzzy Systems

9. Xu Z, Yager R (2006) Some geometric aggregation operators based on intuitionistic fuzzy sets. Int J Gen Syst 35:417-433, 08

10. Xu Z (2007) Some similarity measures of intuitionistic fuzzy sets and their applications to multiple attribute decision making. Fuzzy Optim Decis Making 6:109-121, 06

11. Liu P, Wang Y, Jia F, Fujita H (2020) A multiple attribute decision making three-way model for intuitionistic fuzzy numbers. Int $\mathbf{J}$ Approx Reason 119:177-203, 04

12. Wang $X, X u Z$, Gou $X$ et al (2020) A novel plausible reasoning based on intuitionistic fuzzy propositional logic and its application in decision making. Fuzzy Optimization and Decision Making, $1-24$

13. Garg H, Kumar K (2019) A novel possibility measure to intervalvalued intuitionistic fuzzy set using connection number of set pair analysis and its applications. Neural Computing and Applications, $1-12$

14. Zhou W, Xu Z (2019) Envelopment analysis, preference fusion, and membership improvement of intuitionistic fuzzy numbers. IEEE Transactions on Fuzzy Systems

15. Alcantud JCR, Khameneh AZ, Kilicman A (2020) Aggregation of infinite chains of intuitionistic fuzzy sets and their application to choices with temporal intuitionistic fuzzy information. Inform Sci 514:106-117

16. Ngan RT, Ali M, Tamir DE, Rishe ND, Kandel A et al (2020) Representing complex intuitionistic fuzzy set by quaternion numbers and applications to decision making. Appl Soft Comput 87:105961

17. Song Y, Fu Q, Wang Y-F, Wang X (2019) Divergence-based cross entropy and uncertainty measures of atanassov's intuitionistic fuzzy sets with their application in decision making. Appl Soft Comput 84:105703

18. Son LH, Ngan RT, Ali M, Fujita H, Abdel-Basset M, Giang NL, Manogaran G, Priyan MK (2020) A new representation of intuitionistic fuzzy systems and their applications in critical decision making. IEEE Intell Syst 35(1):6-17

19. Garg H, Singh S (2020) Algorithm for solving group decisionmaking problems based on the similarity measures under type 2 intuitionistic fuzzy sets environment. Soft Comput 24(10):73617381

20. Meng F, Tang J, Fujita H (2019) Linguistic intuitionistic fuzzy preference relations and their application to multi-criteria decision making. Inform Fus 46:77-90, 05

21. W Zhou ZXu (2018) Extended intuitionistic fuzzy sets based on the hesitant fuzzy membership and their application in decision making with risk preference. Int J Intell Syst 33(2):417-443

22. Yager RR (2018) Decision making under measure-based granular uncertainty. Granul Comput 3(4):345-353

23. Wang H, Fang Y-P, Zio E (2019) Risk assessment of an electrical power system considering the influence of traffic congestion on a hypothetical scenario of electrified transportation system in new york stat. IEEE Transactions on Intelligent Transportation Systems

24. Cheong KH, Koh JM (2019) A hybrid genetic-levenberg marquardt algorithm for automated spectrometer design optimization. Ultramicroscopy 202:100-106

25. Deng Y (2020) Uncertainty measure in evidence theory. Sci China Inform Sci 63(11):210201

26. Li Y, Garg H, Deng Y (2020) A new uncertainty measure of discrete Z-numbers. Int J Fuzzy Syst 22:760-776

27. Soo WWM, Cheong KH (2014) Occurrence of complementary processes in parrondo's paradox. Physica A: Stat Mech Applic 412:180-185

28. Li M, Huang S, De Bock J, De Cooman G, Pižurica A (2020) A robust dynamic classifier selection approach for hyperspectral images with imprecise label information. Sensors 20(18):5262

29. Tan Z-X, Koh JM, Koonin EV, Cheong KH (2020) Predator dormancy is a stable adaptive strategy due to parrondo's paradox. Adv Sci 7(3): 1901559

30. Deng X, Jiang W (2019) D number theory based gametheoretic framework in adversarial decision making under a fuzzy environment. Int J Approx Reason 106:194-213

31. Fujita H, Gaeta A, Loia V, Orciuoli F (2019) Resilience analysis of critical infrastructures: a cognitive approach based on granular computing. IEEE Trans Cybern 49(5):1835-1848

32. Pan L, Deng Y (2020) Probability transform based on the ordered weighted averaging and entropy difference. Int J Comput Commun Control 15(4):3743

33. Xiao F (2019) A distance measure for intuitionistic fuzzy sets and its application to pattern classification problems. IEEE Transactions on Systems, Man, and Cybernetics: Systems

34. Atanassov KT, Rangasamy P (1986) Intuitionistic fuzzy sets. Fuzzy Sets Syst 20(1):87-96

35. Yager RR (2020) Mean, variance and covariance of joint measure based uncertain variables. Inform Fus 55:245-250

36. Shafer G (1976) A mathematical theory of evidence, vol 42. Princeton University Press 
37. Mandal P, Ranadive AS (2019) Multi-granulation fuzzy probabilistic rough sets and their corresponding three-way decisions over two universes. Iranian J Fuzzy Syst 16(5):61-76

38. Sivaraman G, Vishnukumar P, Raj MEA (2019) Mcdm based on new membership and non-membership accuracy functions on trapezoidal-valued intuitionistic fuzzy numbers. Soft Comput, $1-11$

39. Garg H, Rani D (2019) Robust averaging-geometric aggregation operators for complex intuitionistic fuzzy sets and their applications to medm process. Arabian Journal for Science and Engineering, 1-17

40. Singh P, Huang Y-P, Wu S (2020) An intuitionistic fuzzy set approach for multi-attribute information classification and decision-making. International Journal of Fuzzy Systems

41. Liu Z, Xiao F (2019) An evidential aggregation method of intuitionistic fuzzy sets based on belief entropy. IEEE Access 7:68905-68916

42. Shu X, Ai Z, Xu Z, Ye J (2019) Integrations of q-rung orthopair fuzzy continuous information. IEEE Trans Fuzzy Syst 27(10):1974-1985

43. Yager RR, Alajlan N, Bazi Y (2018) Aspects of generalized orthopair fuzzy sets. Int J Intell Syst 33(11):2154-2174

44. Li Z, Liu X, Dai J, Chen J, Fujita H (2020) Measures of uncertainty based on gaussian kernel for a fully fuzzy information system. Knowl-Based Syst 196:105791, 03

45. Koh JM, Cheong KH (2019) New doubly-anomalous parrondo's games suggest emergent sustainability and inequality. Nonlin Dyn 96(1):257-266

46. Aydın T, Enginoğlu S (2020) Interval-valued intuitionistic fuzzy parameterized interval-valued intuitionistic fuzzy soft sets and their application in decision-making. Journal of Ambient Intelligence and Humanized Computing, 1-18

47. Cheng C, Xiao F, Cao Z (2019) A new distance for intuitionistic fuzzy sets based on similarity matrix. IEEE Access 7:7043670446

48. Zhang L, Zhan J, Xu Z, Alcantud JCR (2019) Coveringbased general multigranulation intuitionistic fuzzy rough sets and corresponding applications to multi-attribute group decisionmaking. Inform Sci 494:114-140

49. Xue Y, Deng Y (2020) Entailment for Intuitionistic fuzzy sets based on generalized belief structures. Int J Intell Syst 35:963-982

50. Marinov E, Atanassov K (2020) Partially continuous pretopological and topological operators for intuitionistic fuzzy sets. Iranian J Fuzzy Syst 17(2):1-15
51. Feng F, Xu Z, Fujita H, Liang M (2020) Enhancing promethee method with intuitionistic fuzzy soft sets. Int J Intell Syst 35(7):1071-1104

52. Mohtashami M, Eftekhari M (2019) A hybrid filter-based feature selection method via hesitant fuzzy and rough sets concepts. Iranian J Fuzzy Syst 16(2):165-182

Publisher's note Springer Nature remains neutral with regard to jurisdictional claims in published maps and institutional affiliations.

Yong Deng received the Ph.D. degree in Precise Instrumentation from Shanghai Jiao Tong University, Shanghai, China, in 2003. From 2005 to 2011, he was an Associate Professor in the Department of Instrument Science and Technology, Shanghai Jiao Tong University. From 2010, he was a Professor in the School of Computer and Information Science, Southwest University, Chongqing, China. From 2012, he was an Visiting Professor in Vanderbilt University, Nashville, TN, USA. From 2016, he was also a Professor in School of Electronic and Information Engineering, $\mathrm{Xi}$ an Jiaotong University, Xian, China. From 2017, he is the full professor of Institute of Fundamental and Frontier Science, University of Electronic Science and Technology of China, Chengdu, China. He is the JSPS Invitational Fellow in Japan Advanced Institute of Science and Technology, Ishikawa, Japan. Professor Deng has published more than 100 papers in referred journals. His research interests include uncertainty, evidence theory, and complex system modelling. He presents generalized evidence theory, D numbers, Deng entropy and information volume of mass function. He served as the PC member of many conference such as International Conference on Belief Functions. He served as many editorial board members such aseditorial board member of Entropy, Applied Intelligence, Plos One, Defence Science Journal, guest editor of IEEE trans. on fuzzy systems, International Journal of Approximate Reasoning, Sustainability, Mathematical Problems in Engineering, WORK A Journal of Prevention, Assessment \& Rehabilitation, Journal of Systems Science and Systems Engineering, associate editor $\mathrm{f}$ Journal of Organizational and End User Computing. He served as a reviewer for more than 40 SCI-indexed journals. Professor Deng has received numerous honors and awards, including the Elsevier Highly Cited Scientist in China in 2014-2020. 